\title{
Kinetic model of non-isothermal crystal nucleation with transient and athermal effects
}

\author{
Andrzej Ziabicki ${ }^{1}$, Beata Misztal-Faraj ${ }^{1}$, and Leszek Jarecki ${ }^{1, *}$ \\ ${ }^{1}$ Institute of Fundamental Technological Research, Polish Academy of Sciences, Pawinskiego 5 B, Warsaw, Poland
}

Received: 21 March 2016

Accepted: 16 June 2016

Published online:

28 June 2016

(C) The Author(s) 2016. This article is published with open access at Springerlink.com

\begin{abstract}
A kinetic model of primary homogeneous non-isothermal crystal nucleation with transient and athermal effects is developed. For comparison, steady-state and transient isothermal nucleation rates are considered. Kinetic equation for the development of cluster size distribution provides the basis for the model. Transient effects are characterized by the longest relaxation time which increases with temperature at low and moderate undercooling. In isothermal conditions, nucleation rate is controlled by thermal mechanism; in nonisothermal conditions, there appears also athermal mechanism. Closed-form analytical formula for the development of transient cluster size distribution in single-relaxation-time approximation is derived for non-isothermal processes, as well as thermal and athermal nucleation rates and total number of nuclei produced in a cooling or heating run. The transient term contributes to isothermal nucleation kinetics the more the higher is temperature. Under nonisothermal conditions, the relaxation time contributes to the nucleation kinetics by the product with the cooling/heating rate. Considerable transient effects should be expected for the relaxation times as long as $10^{2}-10^{5} \mathrm{~s}$. Contribution of thermal nucleation to the concentration of nuclei is inversely proportional to the temperature rate, while the contribution of athermal nucleation depends on the temperature interval of cooling or heating. Our kinetic model indicates similarities in the nucleation mechanisms in polymers and metals undergoing crystallization. Example computations are presented for molten indium and a linear polymer-polyhydroxybutyrate (PHB). A low-temperature limit is predicted for the nucleation mechanism in $\mathrm{PHB}$, while for indium the mechanism is active in the entire temperature range.
\end{abstract}

Address correspondence to E-mail: ljarecki@ippt.pan.pl 


\section{Introduction}

Crystallization plays important role in material science and technology. Crystal structure, degree of crystallinity, crystal size distribution, and molecular orientation strongly affect mechanical, sorptional, and optical properties of many metals, ceramics, and polymers. Kinetics of the nucleation-controlled crystallization are sensitive to temperature, to temperature history, and to the rate with which the temperature changes during the crystallization time. Optimum temperature and cooling/heating rates must be known whenever desired crystallinity of the material is sought. In variable temperature, nucleation involves different mechanisms responsible for steady-state, transient, and athermal (memory) effects. Kinetics of nucleation have been widely described in books and review articles [1-7]. Whereas steady, isothermal nucleation has been reasonably well studied, the mechanisms involved in variable external conditions (temperature, stress, etc.) still require theoretical and experimental research.

Crystallization involves two successive processes: formation of primary nuclei and crystal growth. Primary nucleation initiates most of the solidification processes in liquids and in solid-solid transformations. The basis of primary crystal nucleation is the formation of atomic or molecular clusters which grow spontaneously to macroscopic dimensions. The nuclei can be formed homogeneously from a pure amorphous phase or by heterogeneous mechanism on foreign particles. The knowledge on the role of the state parameters (temperature, concentration, pressure, stresses) in the nucleation kinetics is important for controlling structure formation in low and high molecular materials. In the early approaches, the thermodynamic criterion of phase instability proposed by Gibbs was used for developing kinetic theory of nucleation in vapors, gasses, and condensed matter [1, 8-12].

In the case of isothermal conditions, the kinetic theories concern steady-state and non-steady-state nucleation. Volmer and Weber [8] originally developed the theory for the condensation of saturated vapors as a process activated by thermal fluctuations and controlled by free energy barrier. The formula for steady-state homogeneous nucleation rate at constant temperature in such systems was derived by Becker and Döring [10], Zeldovich [11], and Frenkel [1] basing on the steady-state cluster volume distribution. With the theory of absolute reaction rates proposed by Eyring [13], homogeneous nucleation rate for condensed systems at constant temperature was derived by Turnbull and Fisher [12]. Spherical kinetic units (atoms) subjected to aggregation into spherical clusters were considered in that model in application to molten metals.

In the classical models of nucleation in condensed phases, various shapes of the aggregating units, nuclei, and clusters were assumed (spheres, cylinders, polyhedra, etc.). Asymmetric kinetic units forming cylindrical clusters [14] or rectangular parallelepipeds [15] were considered in the kinetic theory applied to polymers. For non-spherical clusters, a formal treatment covering cluster volume and shape distribution was proposed by Ziabicki [16]. The kinetic approach to steady-state homogeneous nucleation rate in polymers was verified experimentally by Turnbull et al. [17, 18] and for liquid metals $[19,20]$. In considering kinetics of isothermal nucleation in condensed matter, it is important to account for transient behavior. Kinetic theory of non-steadystate isothermal nucleation controlled by transient distribution of cluster size was considered analytically by Zeldovich [11], Collins [21], Kashchiev [22], Chakraverty [23], Wakeshima [24], Feder [25], Andres [26], and by computer simulations by Kelton [27]. These theories account for a transient time during which the rate of nuclei production approaches steady-state level. In metallic and silicate glasses, the transient time is long enough to be observed experimentally due to the incubation periods representing the time taken for a concentration of nuclei to be achieved [28-31].

The results of computer simulation on transient isothermal nucleation rate and temperature dependence of the time lag in one-component glasses [27] are in best agreement with the Kashchiev theory [22]. The computed time lag is nearly equal to the longest relaxation time of the cluster size distribution, as predicted in [22]. Increasing of the temperature results in strong decrease in the computed time lag from several tens of hours to milliseconds in the temperature range of $\pm 100 \mathrm{~K}$ around the maximum nucleation rate. Basing on the experimental results of Kalinina [32], it was shown by Kelton and Greer [33] that transient isothermal nucleation in lithium disilicate glasses proceeds accordingly to time evolution of cluster distribution from a predetermined initial state, as predicted by the classical theory. 
Using computer simulation, Kelton and Greer [34-36] have predicted that the transient phenomena are important also in non-isothermal homogeneous nucleation (alkali silicates, metallic glasses). The authors have shown that the transient effects reduce nucleation rate the more so, the higher is cooling rate. The transient effects reduce critical cooling rate for achieving glassy state by two to three orders of magnitude, relative to steady-state kinetics, in agreement with the experimental data. Transient effects in non-isothermal nucleation play significant role in the glassy state [31, 37].

For crystallization from metallic liquids, transient effects in nucleation kinetics can play a prominent role at extremely rapid cooling [7] due to very short time lags (relaxation times) in the molten state. At such fast cooling processes, deviation from the steady-state cluster size distribution should be accounted for. Formation of metallic glasses provides an example. It has been shown experimentally [34] that deviation from the steady-state results in reduction in the number of nuclei produced during the quench, scaled with the cooling rate lower by one to tree orders of magnitude than predicted for a steady-state process.

When chemical composition of the nucleating phase differs from that of the mother phase, the effects of long-range diffusion coupled with the attachmentdetachment rates are important in the nucleation kinetics. In the case of one-component systems and significantly asymmetric kinetic units, long-range translational diffusion is coupled with rotational diffusion [38, 39] and may affect transient behavior of isothermal and non-isothermal nucleation.

In the present paper, we derive a simple kinetic model of primary, homogeneous, non-isothermal nucleation. We modify the transient kinetic theory of isothermal nucleation proposed by Kashchiev [22] to obtain a closed-form, analytical expression for the kinetics of transient nucleation in one-component systems under non-isothermal conditions. In our model, the simplest cubic symmetry of the kinetic elements and the longest relaxation time are assumed which allows to analyze the effects considered in this paper. Orientation effects involving non-isodiametric symmetry will be subject of another paper.

The transient non-isothermal nucleation rate is based on time-dependent cluster size distribution controlled by the thermodynamic factors and the longest temperature-dependent relaxation time.
Transient effects with temperature-dependent relaxation time are present also in isothermal conditions. That is why relaxation time appears in the nonisothermal model. Contribution of athermal nucleation in the kinetic model is also accounted for. The concept of athermal nucleation was introduced by Fisher et al. [40], Ziabicki [41], and Kashchiev [5].

We analyze individual factors and mechanisms involved in nucleation kinetics and indicate those processing conditions and material parameters which play decisive role in the overall nucleation rate, in the reference to steady-state and transient isothermal processes. Using our model, numerical evaluation of nucleation kinetics is performed for two example materials: metallic indium and the linear polymer polyhydroxybutyrate (PHB).

\section{Cluster size distribution and nucleation rate}

To describe the formation of atomic or molecular clusters as the basis of crystal nucleation, we follow the classical approach by Volmer and Weber [8], Becker and Döring [10], Turnbull and Fisher [12] and formulate continuous distribution of cluster density $\rho(v)$ in the space of their volumes $v$

$$
\begin{aligned}
& \rho(v)=\frac{1}{V} \frac{\mathrm{dN}(v)}{\mathrm{d} v} \\
& \rho\left(v=v_{0}\right)=\rho_{0} \\
& \rho_{0} v_{0}=\frac{N_{1}}{V}
\end{aligned}
$$

where $v_{0}$ is the volume of a single kinetic unit undergoing aggregation in the system of volume $V$. For condensed systems, we have $\rho_{0}=1 / v_{0}^{2}$.

In our approach with the cluster volume as the size variable, $v$, the continuity equation of the classical kinetic theory of nucleation for cluster size distribution reads

$$
\begin{aligned}
& \frac{\partial \rho(v, t)}{\partial t}+\frac{\partial j(v, t)}{\partial v}=0 \\
& j(v, t)=-D(v, t)\left(\frac{\partial \rho}{\partial v}+\frac{\rho}{k T} \frac{\partial \Delta G(v, t)}{\partial v}\right)
\end{aligned}
$$

Equation (2) describes kinetics of quasi-chemical bimolecular reactions between clusters, $v>v_{0}$, and single kinetic units, $v=v_{0} . D(v, t)$ is a "diffusion coefficient" of clusters in the space of their volumes $v$, and $\Delta G(v, t)$ is free enthalpy of formation a $v$-size 
cluster from the equivalent volume of single elements. The assumed isodiametric geometry makes volume of the molecular clusters, $v$, a single variable of the model. With this variable, basic non-isothermal and transient effects are treated. For cubic kinetic elements assumed in the model, we have $v_{0}=d_{0}^{3} ; v=d^{3}$. Concentration of single kinetic units (atoms, molecules, molecular segments) is assumed to be large, compared to that of dimers, trimers, and higher-order clusters.

Free enthalpy of cluster formation, $\Delta G$, includes a bulk term, $\Delta g$, proportional to undercooling $T_{m}-T$ and a positive term characterized by surface free energy density $\sigma$

$\Delta G(v, T)=\Delta g v+6 \sigma v^{2 / 3}=-\Delta h \frac{\left(T_{m}-T\right)}{T_{m}} v+6 \sigma v^{2 / 3}$

$\Delta h$ is the heat of melting and $T_{m}$ is the equilibrium melting temperature.

Differentiation of $\Delta G$ with respect to $v$ yields critical cluster size $v^{*}$ and the maximum free energy $\Delta G^{*}$ providing thermodynamic barrier for nucleation

$\frac{\mathrm{d} \Delta G}{\mathrm{~d} v}=0 \Rightarrow\left\{\begin{array}{l}v^{*}(T)=\frac{64 \sigma^{3}}{|\Delta g|^{3}}=\frac{64 \sigma^{3} T_{m}^{3}}{\Delta h^{3}\left(T_{m}-T\right)^{3}} \\ \Delta G^{*}(T)=\frac{32 \sigma^{3}}{\Delta g^{2}}=\frac{32 \sigma^{3} T_{m}^{2}}{\Delta h^{2}\left(T_{m}-T\right)^{2}}\end{array}\right.$

Maximum free energy $\Delta G *$ provides potential barrier for the flux of clusters in the space of their volumes $v$. Thermal nucleation rate $\dot{N}$ is defined as the number of overcritical clusters (nuclei) produced in unit time and unit volume of the system

$\dot{N}(T)=j(v *, T)=-D(v *)\left(\frac{\partial \rho}{\partial v}\right)_{v=v *}$

The range of temperatures in which homogeneous thermal nucleation exists is limited. The upper limit is defined by equilibrium melting temperature $T_{m}$

$T=T_{m} \Rightarrow\left\{\begin{array}{l}v^{*}(T) \rightarrow \infty \\ \Delta G^{*}(T) \rightarrow \infty \\ \dot{N}(T)=0\end{array}\right.$

With increasing undercooling, critical cluster size $v^{*}$ decreases. At the lower limiting temperature $T_{\text {lim }}$ $v^{*}$ drops down to the level of a single kinetic unit

$$
\begin{gathered}
T=T_{\lim } \Leftrightarrow v *=v_{0} \\
T_{\lim }=T_{m}\left(1-\frac{4 \sigma}{\Delta h v_{0}^{1 / 3}}\right)
\end{gathered}
$$

With Eq. (7), we introduce the low-temperature bound $T_{\text {lim }}$ for crystallization controlled by homogeneous primary nucleation. Below $T_{\text {lim, }}$ all clusters present in the system and single elements behave like effective nuclei and are able to grow to macroscopic dimensions.

Such a behavior has been analyzed by George [42] as a "nucleative collapse" in the case of high-speed melt spinning. When temperature $T_{\lim }$ is reached, all clusters including monomers become effective nuclei and the kinetics of crystallization is solely controlled by diffusional growth. Evaluation of Eq. (7) using material data from Table 1 yields for PHB $T_{\text {lim }}=359 \mathrm{~K}$ while for indium, $T_{\text {lim }}$ calculated from Eq. (7) is negative. Consequently, primary nucleation mechanism in indium is active in the entire range of temperatures. Non-nucleative temperature range seems to be involved in materials undergoing spinodal decomposition $[43,44]$ where the transition rates are controlled by diffusional growth. Such a behavior may also appear in crystallization of highly oriented polymers $[42,45]$ where orientation entropy strongly reduces the critical cluster size.

Above the critical transition temperature, $T>T_{m}$ the flux of cluster growth disappears and Eq. (2) reduces to

$$
\begin{aligned}
& j_{e q}(v)=0 \\
& \frac{\mathrm{d} \rho_{e q}}{\mathrm{~d} v}+\frac{\rho_{e q}}{k T} \frac{\mathrm{d} \Delta G_{e q}}{\mathrm{~d} v}=0
\end{aligned}
$$

and cluster distribution assumes the Boltzmann form

$$
\begin{aligned}
\rho_{e q}(v, T)= & \rho_{0} \exp \left[\frac{-\Delta G_{e q}(v, T)}{k T}\right] \\
& =\rho_{0} \exp \left[\frac{\Delta h v\left(T_{m}-T\right) / T_{m}-6 \sigma v^{2 / 3}}{k T}\right]
\end{aligned}
$$

Asymptotically, we have

$$
\Delta G_{e q}(v)=\left\{\begin{array}{lll}
0 & \text { for } & v=v_{0} \\
\infty & \text { for } & v \rightarrow \infty
\end{array}\right.
$$

\section{Steady-state isothermal nucleation rate}

To describe steady flow of single kinetic units into the system of clusters and steady flow of large clusters leaving the system, we follow principles of the classical nucleation theory $[8,10,12]$. The boundary conditions in the space of cluster volumes, $v$, include 
constant concentration of single elements and zero concentration of very large clusters

$\rho_{s t}\left(v=v_{0}\right)=\rho_{0}=$ const

$\rho_{s t}\left(v \geq v_{\max }\right)=0$

$v_{\max } \cong 2 v *$

The steady-state model involves an absorbing boundary at $v=v_{\max }$ and a providing boundary at $v=v_{0}$. Without an excessive error, the upper limit $v_{\max }$ can be approximated with double critical size.

In steady-state isothermal conditions, Eq. (2) reduces to

$$
\begin{aligned}
& \frac{\partial \rho_{\mathrm{st}}}{\partial t}=0 \\
& j_{\mathrm{st}}(v)=\mathrm{const} \\
& \frac{\mathrm{d} \rho_{\mathrm{st}}(v, T)}{\mathrm{d} v}+\frac{\rho_{\mathrm{st}}(v, T)}{k T} \frac{\mathrm{d} \Delta G(v, T)}{\mathrm{d} v}=\frac{\operatorname{const}(T)}{D(v, T)}
\end{aligned}
$$

Exact solution of Eq. (12) can be obtained in the form of integrals. In the space of cluster volume, we use, however, a more handy analytical approximation proposed by Zeldovich [11] and Frenkel [1]

$$
\begin{aligned}
\rho_{\mathrm{st}}(v, T) & =\frac{1}{2} \rho_{0} e^{\frac{-\Delta G(v)}{k T}}\{1-\operatorname{erf}[\mathrm{Z}(v-v *)]\} \\
& \frac{\mathrm{d} \rho_{\mathrm{st}}}{\mathrm{d} v}(v, T)=-\rho_{0} e^{\frac{-\Delta G(v)}{k T}} \\
& \times\left[\{1-\operatorname{erf}[Z(v-v *)]\} \frac{1}{2 k T} \frac{\mathrm{d} \Delta G}{\mathrm{~d} v}+\frac{Z}{\sqrt{\pi}} e^{-Z^{2}(v-v *)^{2}}\right]
\end{aligned}
$$

where $Z$ is the half-width of the potential function $\Delta G(v)$, known as the Zeldovich factor

$Z(T)=\sqrt{\left.\frac{-1}{2 k T} \frac{\mathrm{d}^{2} \Delta G}{\mathrm{~d} v^{2}}\right|_{\mho *}}=\frac{\Delta h^{2}\left(T_{m}-T\right)^{2}}{8 \sqrt{6 k T} \sigma^{3 / 2} T_{m}^{2}}$

In the critical point, $v=v^{*}$, steady-state distribution of clusters in the space of cluster volume reduces to

$$
\begin{aligned}
\rho_{\mathrm{st}}(v *) & =\frac{1}{2} \rho_{0} \exp \left[\frac{-32 \sigma^{3} T_{m}^{2}}{k T \Delta h^{2}\left(T_{m}-T\right)^{2}}\right] \\
\left.\frac{\mathrm{d} \rho_{\mathrm{st}}}{\mathrm{d} v}\right|_{v *} & =\frac{-Z}{\sqrt{\pi}} \rho_{0} \exp \left(\frac{-\Delta G^{*}}{k T}\right) \\
& =\frac{-\Delta h^{2}\left(T_{m}-T\right)^{2}}{8 \sqrt{6 \pi k T} \sigma^{3 / 2} T_{m}^{2}} \rho_{0} \exp \left[\frac{-32 \sigma^{3} T_{m}^{2}}{k T \Delta h^{2}\left(T_{m}-T\right)^{2}}\right]
\end{aligned}
$$

The "diffusion coefficient" in the space of cluster volumes, $D(v)$, is proportional to the cluster surface area, $v^{2 / 3}$, and to the frequency of the molecular motions. For the "diffusion coefficient," we assume the Arrhenius approximation with constant transport barrier $E_{D}$, and in case of cubic clusters, we obtain

$D(v, T)=3 v^{2 / 3} v_{0}^{4 / 3} \frac{k T}{h} \exp \left(\frac{-E_{D}}{k T}\right)$

which at $v=v^{*}(T)$ reduces to

$D\left[v^{*}(T), T\right]=\frac{48 \sigma^{2} v_{0}^{4 / 3} T_{m}^{2}}{\Delta h^{2}\left(T_{m}-T\right)^{2}} \frac{k T}{h} \exp \left(\frac{-E_{D}}{k T}\right)$

Thus, we obtain steady-state nucleation rate in the form

$$
\begin{aligned}
& \dot{N}_{s t}(T)=-\left.D\left(v^{*}\right) \frac{d \rho_{s t}}{d v}\right|_{v=v^{*}} \\
&= I_{0} \exp \left(\frac{-E_{D}}{k T}\right) \exp \left[\frac{-32 \sigma^{3} T_{m}^{2}}{k T \Delta h^{2}\left(T_{m}-T\right)^{2}}\right] \\
& I_{0}=v_{0}^{-2 / 3}\left(\frac{6 \sigma k T}{\pi h^{2}}\right)^{1 / 2}
\end{aligned}
$$

\section{Transient isothermal nucleation rate}

In any real system, the distribution of clusters is subject to molecular rearrangement, and steady state is reached only asymptotically. Transient isothermal solutions of the continuity equation, Eq. (2), have been obtained by Collins [21], Kashchiev [22], Chakraverty [23], and others. For the cluster size distribution in the space of their volumes, separation of variables in Eq. (2) yields an infinite sum of exponentials

$\rho(v, t)=a_{0}(v)+\sum_{i=1}^{\infty} a_{i}(v) \exp \left(-t / \tau_{i}\right)$

where $\tau_{i}$ denotes relaxation times and $a_{i}$ are $v$-dependent parameters.

We approximate the distribution, Eq. (19), by a single time-dependent term with the longest relaxation time $\tau=\tau_{1}$

$\rho(v, t) \cong a_{0}(v)+a_{1}(v) \exp (-t / \tau)$

With the boundary conditions

$$
\begin{aligned}
& \rho(v, t=0)=\rho_{\text {in }}(v) \\
& \rho(v, t \rightarrow \infty)=\rho_{s t}(v)
\end{aligned}
$$

we obtain the following distribution controlled by single-relaxation time

$$
\rho(v, t) \cong \rho_{\mathrm{st}}(v)+\left[\rho_{\mathrm{in}}(v)-\rho_{\mathrm{st}}(v)\right] \exp (-t / \tau)
$$


where $\tau$ is a temperature-dependent material characteristics. The nucleation time lag $\theta$ is expressed by the longest relaxation time $\tau$ by the formula $\theta=\pi^{2} \tau / 6$ [22]. It is evident that at infinitely long $\tau$, the initial distribution $\rho_{\text {in }}(v)$ remains unchanged, and at $\tau=0$, steady state is reached instantaneously.

By differentiation of Eq. (22), we obtain the following equation for the rate of variation in the distribution in the processes controlled by the longest relaxation time

$\frac{\partial \rho(v, t)}{\partial t}=\frac{\rho_{\mathrm{st}}(v)-\rho(v, t)}{\tau}$

The rate of variation is proportional to the difference between the asymptotic steady-state distribution and the instantaneous distribution, as well as inversely proportional to the temperature-dependent relaxation time $\tau$. This relationship will be used below in this paper for determining the development of cluster size distribution in non-isothermal processes, by integrating Eq. (23) with $\tau$ and $\rho_{s t}(v)$ dependent on varying temperature.

Application of Eqs. (5), (18), and (22) yields isothermal transient nucleation rate

$$
\begin{aligned}
\dot{N}_{\text {trans }}(t) & =-D(v *)\left[\left.\frac{\partial \rho_{s t}}{\partial v}\right|_{v *}+\left(\left.\frac{\partial \rho_{\text {in }}}{\partial v}\right|_{v *}-\left.\frac{\partial \rho_{s t}}{\partial v}\right|_{v *}\right) \exp (-t / \tau)\right] \\
& =\dot{N}_{\text {st }}+\left(\dot{N}_{\text {in }}-\dot{N}_{\text {st }}\right) \exp (-t / \tau)
\end{aligned}
$$

where $\dot{N}_{\text {in }}$ and $\dot{N}_{s t}$ are nucleation rates corresponding, respectively, to the initial and steady-state cluster distributions. The relaxation time of transient nucleation rate is identical with the relaxation time of cluster size distribution $\tau$.

We consider amorphous melt superheated to the temperature $T_{0}>T_{m}$ and rapidly quenched to a constant crystallization temperature $T<T_{m}$. Nucleation proceeds in the temperature $T$ starting with an initial cluster size distribution in the melt equal to the equilibrium Boltzmann distribution $\rho_{\text {in }}(v, T)=$ $\rho_{\text {eq }}\left(v, T_{0}\right)$ at $T_{0}>T_{m}$. The initial nucleation rate $\dot{N}_{\text {in }}$ at $T$ resulting from the equilibrium cluster distribution quenched from the overheating temperature $T_{0}$ reads

$$
\dot{N}_{\text {in }}(T)=-\left.D\left[v^{*}(T), T\right] \frac{\partial \rho_{e q}\left(v, T_{0}\right)}{\partial v}\right|_{v *(T)}
$$

From Eq. (9), we have $\left.\frac{\partial \rho_{e q}\left(v, T_{0}\right)}{\partial v}\right|_{v^{*}(T)}=-\rho_{0} \exp \left[-\frac{\Delta G\left(v^{*}(T), T_{0}\right)}{k T_{0}}\right] \frac{\Delta h\left(T_{0}-T\right)}{k T_{0} T_{m}}$

while the steady-state nucleation rate $\dot{N}_{s t}(T)$ is given by Eq. (18). The half-time $t_{1 / 2}(T)$ at which isothermal transient nucleation rate reaches $50 \%$ of the steadystate value reads

$\frac{t_{1 / 2}}{\tau}=\ln \left[2\left(1-\frac{\dot{N}_{\text {in }}}{\dot{N}_{\text {st }}}\right)\right]$

Kashchiev [22] and Chakraverty [23] obtained the longest relaxation time in the form

$\tau(T)=\frac{1}{2 D\left[v^{*}(T)\right] Z^{2}(T)}$

where $D(v *)$ and the Zeldovich factor $Z(T)$ are given by Eqs. (17) and (14), respectively. The relaxation time $\tau$ represents time of jumping of a critical-size cluster $v^{*}$ over the thermodynamic barrier $Z$ and is expressed by the following function of temperature

$\tau(T)=\frac{192 k T \sigma^{3}}{D(v *, T) \Delta g^{4}(T)}=\frac{4 \sigma T_{m}^{2}}{\Delta h^{2}\left(T_{m}-T\right)^{2} v_{0}^{4 / 3}} h \exp \left(\frac{E_{D}}{k T}\right)$

$\tau$ is controlled by thermodynamic driving force $\Delta g(T)$ and the coefficient $D(T)$.

\section{Nucleation in non-isothermal conditions}

Experimental observations and computer simulations indicate that time variation in thermodynamic parameters plays significant role in the nucleation kinetics. Continuity equation, Eq. (2), with time-dependent "diffusion coefficient" $D$ and free enthalpy of cluster formation $\Delta G$ cannot be solved by separation of the variables. For a semiquantitative analysis of non-isothermal primary homogeneous nucleation, we consider an approximate single-relaxation-time model for time development of the cluster size distribution using temperature-dependent functions $\rho_{\text {st }}(T)$ and $\tau(T)$ in Eq. (23).

\section{Transient non-isothermal nucleation rate}

Consider temperature $T$ monotonically varying in time in the range $T<T_{m}$ with the rate $\dot{T}=\mathrm{d} T / \mathrm{d} t$ from $T_{i n}$. Replacing time variable with temperature 
$T(t)$ in Eq. (23), we obtain the following formula for variation in the transient cluster size distribution $\rho(v, T)$ with temperature under non-isothermal conditions

$\dot{T}(T) \frac{\partial \rho(v, T)}{\partial T}=\frac{\rho_{\mathrm{st}}(v, T)-\rho(v, T)}{\tau(T)}$

controlled by the relaxation time $\tau(T)$ and steadystate limit $\rho_{\mathrm{st}}(v, T)$ of the distribution. For nonisothermal processes, the initial cluster size distribution at the start, $\rho_{\text {in }}(v)$, is assumed as steady-state distribution at the initial temperature $T_{\text {in }}$ which is below $T_{m}$

$$
t=0
$$

$T=T_{\text {in }}<T_{m}$

$\rho=\rho_{\text {in }}(v)=\rho_{\text {st }}\left(v, T_{\text {in }}\right)$

By integration of Eq. (30) with time (temperature-)dependent material functions and boundary conditions from Eq. (31), we obtain the following formula for temperature-dependent cluster size distribution with transient effects under cooling or heating

$$
\begin{aligned}
& \rho_{\text {trans }}(v, T)=\exp \left(-\int_{T_{\text {in }}}^{T} \frac{\mathrm{d} T^{\prime}}{\dot{T}\left(T^{\prime}\right) \tau\left(T^{\prime}\right)}\right) \\
& \times\left[\rho_{\text {in }}(v)+\int_{T_{\text {in }}}^{T} \exp \left(+\int_{T_{\text {in }}}^{T^{\prime}} \frac{\mathrm{d} T^{\prime \prime}}{\dot{T}\left(T^{\prime \prime}\right) \tau\left(T^{\prime \prime}\right)}\right) \frac{\rho_{s t}\left(v, T^{\prime}\right)}{\dot{T}\left(T^{\prime}\right) \tau\left(T^{\prime}\right)} \mathrm{d} T^{\prime}\right]
\end{aligned}
$$

The transient effects in the cluster distribution under non-isothermal conditions, Eq. (32), are controlled by the product of the relaxation time and cooling/heating rate during the process, $\dot{T}(T) \tau(T)$, while under isothermal conditions discussed, they are controlled solely by $\tau(T)$.

Asymptotic behavior of the transient cluster distribution in non-isothermal conditions can be characterized as follows
Differentiation of the distribution terms in Eq. (32) with respect to cluster volume leads us to the following original formula for transient nucleation rate under non-isothermal conditions where the critical size $v^{*}$ and the coefficient $D(v *)$ refer to instantaneous temperature $T$

$$
\begin{aligned}
& \dot{N}_{\text {trans }}(T)=-D(T, v *)\left[\left.\frac{\partial \rho_{\text {in }}(v)}{\partial v}\right|_{v *(T)} \exp \left(-\int_{T_{\text {in }}}^{T} \frac{\mathrm{d} T^{\prime}}{\dot{T}\left(T^{\prime}\right) \tau\left(T^{\prime}\right)}\right)\right. \\
& \left.+.\left.\int_{T_{\text {in }}}^{T} \frac{\partial \rho_{\text {st }}\left(v, T^{\prime}\right)}{\partial v}\right|_{v *(T)} \exp \left(-\int_{T^{\prime}}^{T} \frac{\mathrm{d} T^{\prime \prime}}{\dot{T}\left(T^{\prime \prime}\right) \tau\left(T^{\prime \prime}\right)}\right) \frac{\mathrm{d} T^{\prime}}{\dot{T}\left(T^{\prime}\right) \tau\left(T^{\prime}\right)}\right]
\end{aligned}
$$

The initial distribution at the start, $\rho_{\text {in }}(v)$, in Eq. (34) is a steady-state distribution given by Eq. (13) at $T_{\text {in }}<T_{m}$.

Consider the process in which cooling (heating) rate $\dot{T}$ is a constant and the relaxation time $\tau$ varies with temperature according to Eq. (29). Then, the instantaneous nucleation rate at $T$ reduces to

$$
\begin{aligned}
& \dot{N}_{\text {trans }}(T, \dot{T})=-D(T, v *) \\
& \quad \times\left[\left.\frac{\partial \rho_{\text {in }}(v)}{\partial v}\right|_{v *(T)} \exp \left(-\frac{1}{\dot{T}} \int_{T_{\text {in }}}^{T} \frac{\mathrm{d} T^{\prime}}{\tau\left(T^{\prime}\right)}\right)\right. \\
& \left.\quad+\left.\frac{1}{\dot{T}} \int_{T_{\text {in }}}^{T} \frac{\partial \rho_{s t}\left(v, T^{\prime}\right)}{\partial v}\right|_{v *(T)} \exp \left(-\frac{1}{\dot{T}} \int_{T^{\prime}}^{T} \frac{\mathrm{d} T^{\prime \prime}}{\tau\left(T^{\prime \prime}\right)}\right) \frac{\mathrm{d} T^{\prime}}{\tau\left(T^{\prime}\right)}\right]
\end{aligned}
$$

Nucleation rate in transient conditions is a function of three factors: undercooling $T_{m}-T$, temperaturedependent relaxation time, $\tau(T)$, and cooling/heating rate, $\dot{T}$. The relaxation time $\tau$ and cooling rate $\dot{T}$ affect nucleation kinetics in a similar way. The longer is $\tau$ and / or the faster is cooling, the closer is nucleation rate to the initial value. On the other hand, slow cooling rates and/or short relaxation times move nucleation rate in the direction of asymptotic steady state.

$$
\rho_{\text {trans }}(v, T)=\left\{\begin{array}{lll}
\tau=0 ; \dot{T} \neq 0 & \rho=\rho_{\text {st }}[v, T(t)] & \text { quasi - static } \\
\tau=0 ; \dot{T}=0 & \rho=\rho_{\text {st }}[v, T] & \text { isothermal, steady - state } \\
\tau \rightarrow \infty ; \operatorname{any} \dot{T} & \rho=\rho_{\text {in }}(v) & \text { quenched initial } \\
|\dot{T}| \rightarrow \infty ; \operatorname{any} \tau & \rho=\rho_{\text {in }}(v) & \text { quenched initial }
\end{array}\right\}
$$




\section{Thermal versus athermal nucleation}

Besides thermal nucleation discusses above, under non-isothermal conditions there appears also another mechanism, athermal nucleation. The total concentration of supercritical clusters in a system is related to the cluster size distribution $\rho(v, t)$

$N^{*}(t)=\int_{v *(t)}^{\infty} \rho(v, t) \mathrm{d} v$

The rate with which $N^{*}$ is changed, i.e., nucleation rate, results from the differentiation of the integral in Eq. (36) with respect to time

$$
\begin{gathered}
\dot{N}(t)=\frac{\mathrm{d}}{\mathrm{d} t} \int_{v *(t)}^{\infty} \rho(v, t) \mathrm{d} v=\int_{v *(t)}^{\infty} \frac{\partial \rho(v, t)}{\partial t} \mathrm{~d} v-\frac{\mathrm{d} v^{*}}{\mathrm{~d} t} \rho\left(v^{*}, t\right) \\
\int_{v *(t)}^{\infty} \frac{\partial \rho(v, t)}{\partial t} \mathrm{~d} v=j\left(v^{*}\right)-j(\infty)=j\left(v^{*}\right)=\dot{N}_{\mathrm{th}}(t) \\
\dot{N}_{\text {ath }}(t)=-\frac{\mathrm{d} v^{*}}{\mathrm{~d} t} \rho\left(v^{*}, t\right)
\end{gathered}
$$

Differentiation of the kernel followed by the application of the Gauss-Ostrogradsky theorem yields flux of clusters leaving the subcritical region, $v<v^{*}$, and entering the region of stable nuclei, $v>v^{*}$. This mechanism is known as thermal nucleation. In steady-state isothermal conditions, the flux term $j\left(v^{*}\right)$ reduces to $\dot{N}_{s t}(T)$, Eq. (18). In variable external conditions, when critical cluster size $v^{*}$ changes in time, there appears another term in Eq. (37) resulting from the differentiation of the lower integration limit, $v^{*}(t)$. This term describes athermal nucleation which is proportional to the rate of external conditions (temperature, $T$, pressure, $p$, etc.)

$$
\begin{aligned}
\dot{N}_{\text {ath }}(t) & =-\frac{\mathrm{d} v^{*}}{\mathrm{~d} t} \rho\left(v^{*}, t\right) \\
& =-\left[\frac{\partial v *}{\partial T} \dot{T}+\frac{\partial v *}{\partial p} \dot{p}+\cdots\right] \rho\left(v^{*}, t\right)
\end{aligned}
$$

Depending on the sign of the derivative $\mathrm{d} v^{*} / \mathrm{d} t$, the athermal process may increase or reduce the number of nuclei in the system, e.g., cooling $(\dot{T}<0)$ and/or compression $(\dot{p}>0)$ virtually produces new nuclei, while heating or decompression associated with an increase in $v^{*}$ makes some nuclei lose their stability. In the latter conditions, the athermal nucleation rate is negative. It should be mentioned that finite critical cluster size is defined only in the conditions of undercooling. Above the equilibrium transition temperature, $T>T_{m}$, the critical cluster size is infinite and no nucleation, thermal or athermal, is possible

$\dot{N}_{\text {ath }}(T)=\left\{\begin{array}{cl}>0 & T<T_{m} ; \dot{T}<0 \\ <0 & T<T_{m} ; \dot{T}>0 \\ =0 & T=T_{m} \\ =0 & T>T_{m}\end{array}\right.$

The concept of athermal nucleation has originally been introduced by Fisher, Hollomon, and Turnbull [40] and later derived in the form of Eq. (37) by Ziabicki [41] and Kashchiev [22]. Athermal nucleation is not controlled by diffusion and does not require thermal activation. Clusters acquire the status of nuclei under the change in external conditions. Reduction in temperature [46], or increase in pressure [47] shifts the criterion of cluster stability to smaller critical volume $v^{*}$, thus yielding virtual production of stable nuclei. In other words, some clusters, unstable in the conditions $\left(T_{1}, p_{1}\right)$, are promoted to the rank of nuclei by changing thermodynamic state of the system to $\left(T_{2}, p_{2}\right)$

$$
\begin{gathered}
T_{1} ; v<v^{*}\left(T_{1}\right) \stackrel{\text { cooling }}{\longrightarrow} T_{2}<T_{1} ; v>v^{*}\left(T_{2}\right) \\
p_{1} ; v<v^{*}\left(p_{1}\right) \stackrel{\text { compression }}{\longrightarrow} p_{2}>p_{1} ; v>v^{*}\left(p_{2}\right)
\end{gathered}
$$

In the conditions of cooling and steady-state cluster distribution, $\rho_{\mathrm{st}}(v)$, we obtain the following formula for athermal nucleation rate from Eqs. (15) and (37)

$$
\begin{aligned}
\dot{N}_{\text {ath }}(T, \dot{T}) & =-\dot{T} \rho_{\text {st }}\left(v^{*}\right) \frac{\mathrm{d} v^{*}}{\mathrm{~d} T}=-\dot{T} \Phi(T) \\
\Phi(T) & =\frac{96 \sigma^{3} T_{m}^{3}}{v_{0}^{2} \Delta h^{3}\left(T_{m}-T\right)^{4}} \exp \left[\frac{-32 \sigma^{3} T_{m}^{2}}{k T \Delta h^{2}\left(T_{m}-T\right)^{2}}\right]
\end{aligned}
$$

Replacing $\rho_{s t}(v)$ in Eq. (41) by transient cluster size distribution $\rho_{\text {trans }}(v, T)$ given by Eq. (32), an analytical closed-form expression for athermal nucleation rate is obtained, accounting for the relaxation effects.

It should be emphasized that both thermal and athermal nuclei are homogeneous by nature and physically undistinguishable. Equation (41) accounts for athermal effects in sporadic homogeneous nucleation, without predetermined homogeneous nucleation. To account for the effects of predetermined nucleation, for example memory effects, the distribution $\rho_{\mathrm{st}}\left(v^{*}\right)$ in Eq. (41) should be modified for the predetermined clusters contribution, what is not a subject of this paper. 


\section{Concentration of nuclei produced in a single cooling/heating run}

The factor subsequent to nucleation which controls crystallization is the concentration of nuclei produced during cooling or heating. For steady-state isothermal processes, concentration of thermal nuclei produced within the time interval $\Delta t$ is

$\Delta N_{\text {st }}(\Delta t)=\dot{N}_{\text {st }}(T) \cdot \Delta t$

Consider a single cooling/heating run starting at $T_{0}<T_{m}$. With transient effects in non-isothermal process, we obtain the following closed-form analytical formula for the concentration of nuclei produced in the temperature interval $\left(T_{0}, T\right)$

$\Delta N(T)=\int_{T_{0}}^{T} \frac{\dot{N}_{\text {trans }}\left(T^{\prime}, \dot{T}\right) \mathrm{d} T^{\prime}}{\dot{T}\left(T^{\prime}\right)}$

where $\dot{N}_{\text {trans }}\left(T^{\prime}, \dot{T}\right)$ is given by Eq. (35).

In the nucleation considered as a quasi-static process (steady-state nucleation rate instantaneously follows varying temperature), concentration of nuclei produced at constant $\dot{T}$, at the absence of transient and athermal effects, is expressed by the integral

$\Delta N_{\mathrm{qs}}(T)=\frac{1}{\dot{T}} \int_{T_{0}}^{T} \dot{N}_{\mathrm{st}}\left(T^{\prime}\right) \mathrm{d} T^{\prime}$

$\dot{N}_{s t}(T)$ is a positive material function, and the concentration of nuclei produced in a quasi-static run is controlled by the temperature interval and the cooling/heating rate. For both cooling and heating, concentration of nuclei produced in the transient or quasi-static processes increases, Eqs. (43) and (44). Cooling or heating below $T_{m}$ provides positive contribution to the concentration of thermal nuclei.

Different behavior is found for athermal nucleation. Integrating the athermal nucleation rate in the quasi-static limit, Eq. (41), concentration of athermal nuclei produced in the run is obtained, independently of the cooling/heating rate $\dot{T}$

$\Delta N_{\text {ath }}(T)=-\int_{T_{0}}^{T} \Phi\left(T^{\prime}\right) \mathrm{d} T^{\prime}$

Unlike for thermal nucleation, contribution of athermal process is different for cooling and heating. Under cooling, athermal nuclei are produced,
$\Delta N_{\text {ath }}(T)>0$, while heating results in reduction in the nuclei concentration, $\Delta N_{\text {ath }}(T)<0$.

Total concentration of nuclei at $T$ created in the system during a single cooling/heating run in quasistatic process is expressed by the sum

$$
\begin{aligned}
\Delta N(T) & =\Delta N_{\mathrm{qs}}(T)+\Delta N_{\mathrm{ath}}(T) \\
& =\int_{T_{0}}^{T} \frac{\dot{N}_{\mathrm{st}}\left(T^{\prime}\right) \mathrm{d} T^{\prime}}{\dot{T}}-\int_{T_{0}}^{T} \Phi\left(T^{\prime}\right) \mathrm{d} T^{\prime}
\end{aligned}
$$

It is evident that the thermal contribution, $\Delta N_{\mathrm{qs}}$, is inversely proportional to the cooling/heating rate, while the athermal contribution depends only on the temperature interval $\left(T_{0}, T\right)$.

\section{Discussion of the results}

The model in single-relaxation-time approximation allows to predict transient nucleation rate under isothermal and non-isothermal conditions, athermal nucleation kinetics, as well as influence of individual material and processing parameters and concentration of nuclei produced in a cooling or heating run. Concentration of nuclei produced in a single cooling/ heating run is controlled by initial and target temperature and the cooling/heating rate. The effect of temperature is associated with the driving force represented by the product of heat of melting and the degree of undercooling. Cooling rate renormalizes timescale into temperature (Eq. 43) and affects transient thermal nucleation rate through the product with the relaxation time (Eq. 34). Transient effects present in isothermal and non-isothermal processes are associated with activation energy via the relaxation time.

Variation in cluster size distribution provides a basis for nucleation rate and is assumed to be controlled by the longest relaxation time. Thermodynamic driving force is considered as a linear function of undercooling; molecular transport effects are described by the Arrhenius formula with a constant activation energy $E_{D}$. The range of temperatures available for thermal nucleation is limited to $T_{\lim } \leq T<T_{m}$ for PHB. When temperature $T_{\lim }$ is reached, all clusters including monomers become effective nuclei and the kinetics of crystallization is solely controlled by diffusional growth. For indium, the nucleation mechanism is active in the entire range 
of temperatures due to much smaller molecular volume of the kinetic units.

Besides thermal nucleation mechanism, there appears athermal nucleation. The thermal and athermal nuclei are homogeneous by nature and physically undistinguishable. Athermal nuclei do no require thermal activation of molecular transport because they are created due to a change in the critical cluster size under changing temperature.

Isothermal nucleation rate in this model with a single-relaxation time consists of a steady-state and transient terms. Figure 1 shows steady-state nucleation rate $\dot{N}_{s t}$ versus temperature $T$ calculated from Eq. (18) for indium and Fig. 2 the same characteristics for PHB. Thermodynamic barrier to nucleation, $\Delta G^{*}$, approaches infinity at $T=T_{m}$ and determines upper limit of nucleation. With increased undercooling, $\Delta G^{*}$ is reduced and the thermodynamic driving force increases. At the same time, cooling suppresses molecular mobility and slows nucleation down. Competition between the thermodynamic and transport effects leads to a maximum of $\dot{N}_{s t}(T)$ at $T=T_{\max }$. The calculated temperature $T_{\max }$ for PHB is $288 \mathrm{~K}$, i.e., lies beyond the range of nucleation, Eq. (7). Virtual continuation of the function $\dot{N}_{s t}(T)$ below $T_{\lim }$ is presented as a dashed curve in Fig. 2. For indium, $T_{\max }=162 \mathrm{~K}$ and lies within the range of nucleation.

Nucleation rates, very small in the vicinity of melting temperature (Figs. 1, 2), increase with increasing undercooling. $\dot{N}_{s t}(T)$ for the investigated materials covers wide range of nucleation rates from zero to $10^{22}-10^{26} \mathrm{~cm}^{-3} \mathrm{~s}^{-1}$. The pre-exponential factor $I_{0}$ calculated from Eq. (18) is a weak function of

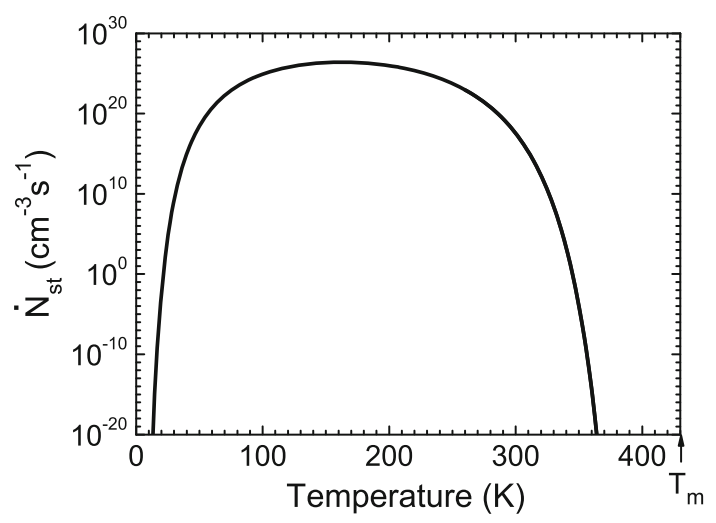

Figure 1 Steady-state nucleation rate $\dot{N}_{\text {st }}$ for indium versus temperature $T . T_{m}$-equilibrium melting temperature. temperature. For indium, the calculated value of $I_{0}$ slightly increases from $2.37 \times 10^{35} \mathrm{~cm}^{-3} \mathrm{~s}^{-1}$ at $280 \mathrm{~K}$ to $2.83 \times 10^{35} \mathrm{~cm}^{-3} \mathrm{~s}^{-1}$ at $400 \mathrm{~K}$. This is well consistent with the average value $I_{0} \approx 10^{35 \pm 1} \mathrm{~cm}^{-3} \mathrm{~s}^{-1}$ reported from experimental investigations on liquid metals [19]. For PHB, the calculated factor $I_{0}$ amounts to $0.87 \times 10^{34} \mathrm{~cm}^{-3} \mathrm{~s}^{-1}$ at $350 \mathrm{~K}$ and $1.11 \times 10^{34} \mathrm{~cm}^{-3} \mathrm{~s}^{-1}$ at $440 \mathrm{~K}$. Experimental data on nucleation for polymers analyzed by Hoffman [3] yield $I_{0}$ of the same order, i.e., $10^{34} \mathrm{~cm}^{-3} \mathrm{~s}^{-1}$.

Figures 3 and 4 show transient isothermal nucleation rates reduced by their steady-state values, $\dot{N}_{\text {trans }}(t) / \dot{N}_{\text {st }}$, calculated from Eqs. (24) and (18) versus time $t$ for indium and PHB. The plots are shown for different temperatures $T$ and corresponding nucleation relaxation times $\tau$, calculated with the initial equilibrium Boltzmann distribution of clusters quenched to $T$ from $T_{0}=T_{m}+10 \mathrm{~K}$. The isothermal transient nucleation rate starts from a very low level, $\dot{N}_{\text {in }} \cong 0$, determined by the quenched initial cluster distribution from $T_{0}$ and increases in time asymptotically approaching steady-state level $\dot{N}_{\text {st }}$. The plots for indium and PHB are shown for three nucleation temperatures and corresponding nucleation relaxation times. The higher is nucleation temperature and longer is the relaxation time, the more nucleation rate does lag behind the steady-state value.

Figure 5 illustrates temperature dependence of the reduced halftime of isothermal transient nucleation rate, $t_{1 / 2} / \tau$, calculated from Eq. (27) for indium and PHB. For each material, the plots for various overheating temperatures $T_{0}$ overlap. In the range of high temperatures (above $200 \mathrm{~K}$ for indium and above $T_{\mathrm{lim}}$ for PHB), the reduced halftime is nearly $\ln 2$ which means that in this temperature range, contribution of the initial cluster distribution quenched from $T_{0}$ above $T_{m}$ is negligible. In lower temperatures, the halftime for indium rapidly drops to zero because the initial nucleation rate resulting from the quenched distribution tends to one half of the steady-state value, Eq. (27). For PHB, variation in the reduced halftime in the low-temperature range is beyond the nucleation mode.

Under non-isothermal conditions, thermal nucleation rate is influenced by the product of cooling rate and temperature-dependent relaxation time, $\dot{T} \tau$. Nucleation rate is reduced by the relaxation effects the more, the longer is relaxation time and the faster is cooling. Under slow cooling and/or short 


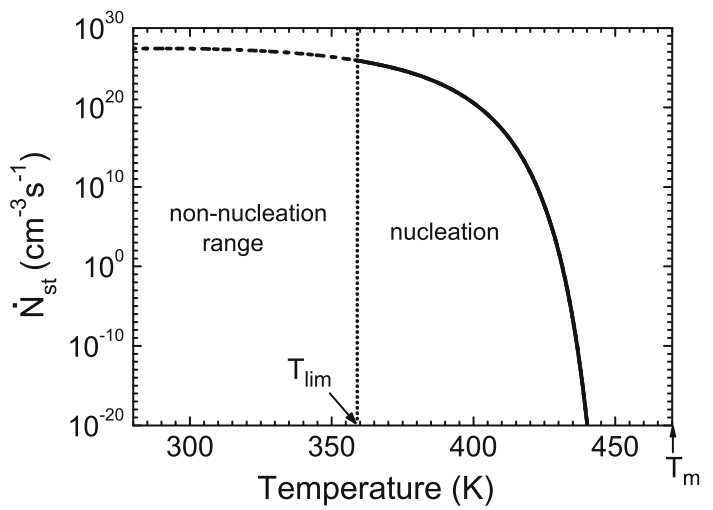

Figure 2 Steady-state nucleation rate $\dot{N}_{\text {st }}$ for PHB versus temperature $T$ in the range well above glass transition. $T_{m}$-equilibrium melting temperature, $T_{\text {lim }}$-limiting temperature for nucleation. The plot in the non-nucleation range indicated by dashed line.

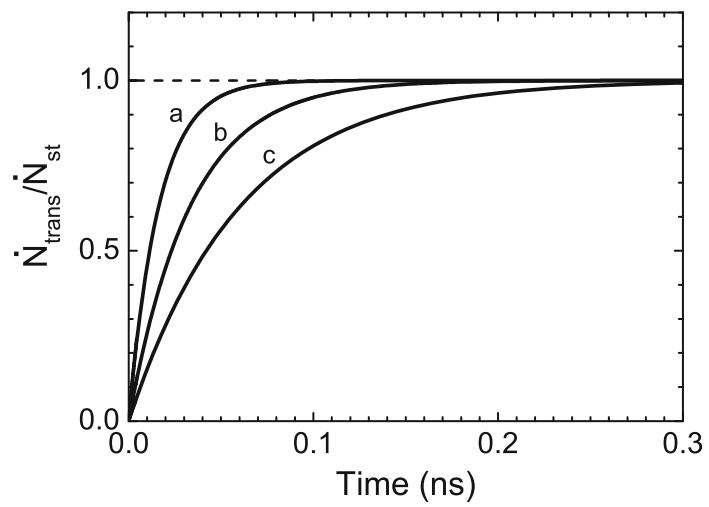

Figure 3 Reduced isothermal transient nucleation rates $\dot{N}_{\text {trans }} / \dot{N}_{\text {st }}$ versus time $t$ for indium. a $T=320 \mathrm{~K}, \tau=0.016 \mathrm{~ns} ; \quad b$ $T=360 \mathrm{~K}, \tau=0.033 \mathrm{~ns} ; \mathrm{c} T=380 \mathrm{~K}, \tau=0.061 \mathrm{~ns}$. Rapid quench to $T$ from $T_{0}=T_{m}+10 \mathrm{~K}$.

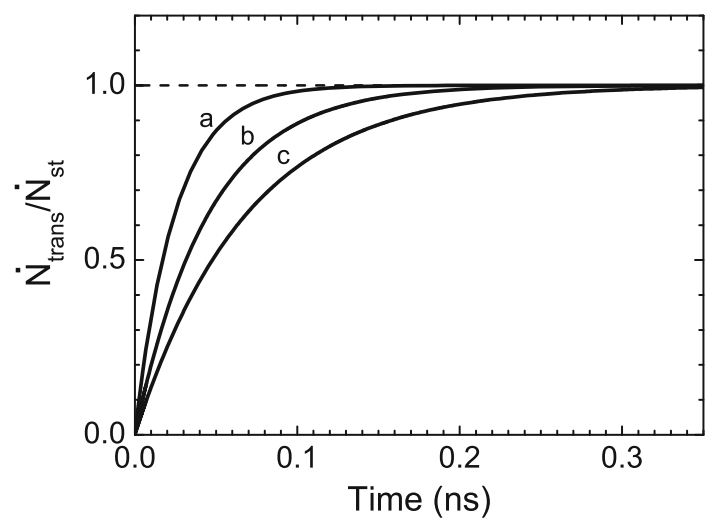

Figure 4 Reduced isothermal transient nucleation rate $\dot{N}_{\text {trans }} / \dot{N}_{s t}$ versus time $t$ for PHB. a $T=400 \mathrm{~K}, \tau=0.025 \mathrm{~ns}$; b $T=430 \mathrm{~K}$, $\tau=0.045 \mathrm{~ns} ;$ c $T=440 \mathrm{~K}, \tau=0.069 \mathrm{~ns}$. Rapid quench to $T$ from $T_{0}=T_{m}+10 \mathrm{~K}$.
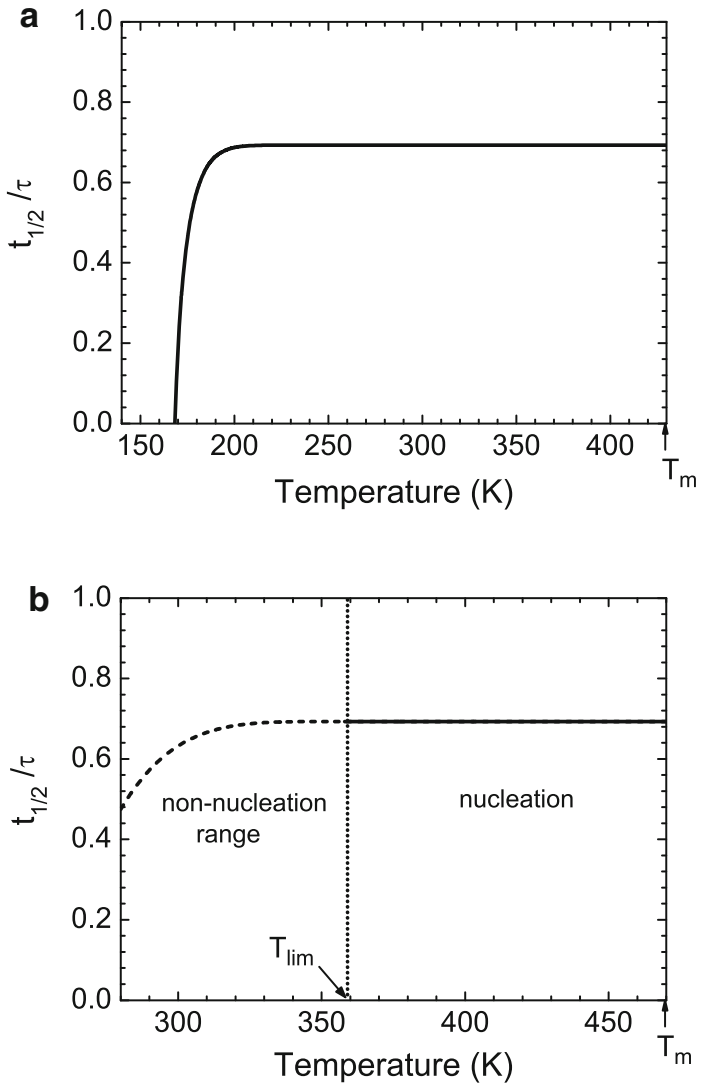

Figure 5 Reduced halftime of the transient nucleation rate $t_{1 / 2} / \tau$ versus $T$ calculated for- $\mathbf{a}$ indium, $\mathbf{b}$ PHB. Rapid quench from the overheating $T_{0}-T_{m}=10,30,50 \mathrm{~K}$ (the plots overlap). Dashed plot - the non-nucleation range.

relaxation times, nucleation rate approaches asymptotically steady state. Temperature dependence of the relaxation time $\tau$ and transient nucleation rates for different values of the product $\dot{T} \tau$ computed for indium and PHB from Eqs. (29) and (35) are presented in Fig. 6. In the melting temperature $T=T_{m}$, $\Delta g$ is zero and the relaxation time is infinitely long. The function $\tau(T)$ exhibits a minimum and increases in the range of large undercoolings. The shortest relaxation time calculated for indium, $\tau=0.009 \mathrm{~ns}$, is found to appear at $230 \mathrm{~K}$ and that for $\mathrm{PHB}$, $\tau=0.020 \mathrm{~ns}$, at $374 \mathrm{~K}$.

Scanty literature data on the relaxation time in primary crystal nucleation cover wide range of values [5] including subnanosecond values calculated from Eq. (29) for liquid indium and molten PHB. Processes controlled by so short relaxation times seem to have no effect on crystal nucleation in liquidto-solid transitions. On the other hand, crystallization from the solid or glassy state involves relaxation 

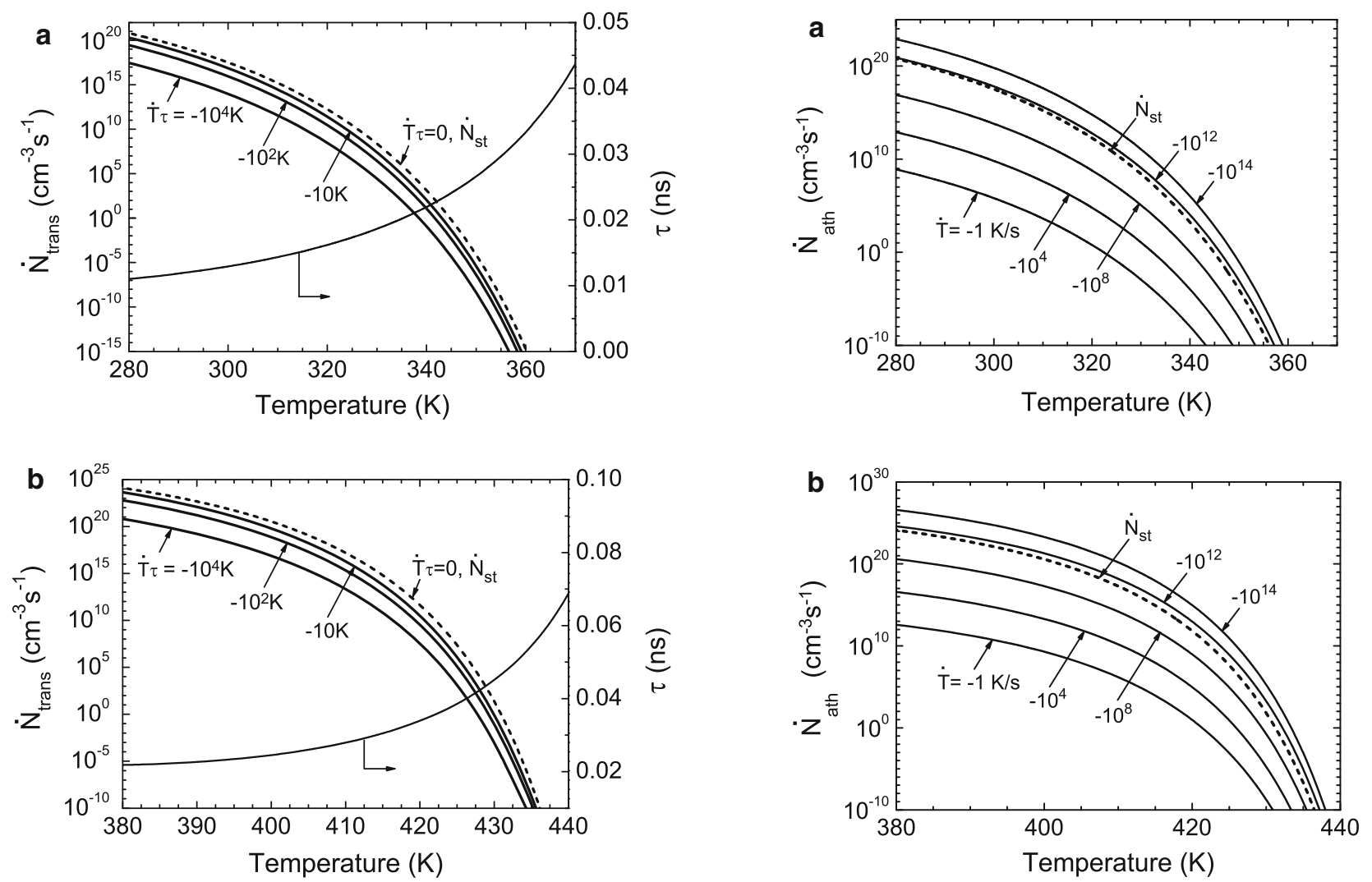

Figure 6 Non-isothermal transient nucleation rates $\dot{N}_{\text {trans }}$ and relaxation time $\tau$ versus temperature $T$ for $-\mathbf{a}$ indium, $\mathbf{b}$ PHB. Parameter $\dot{T} \tau$ indicated. Initial temperature of non-isothermal nucleation $T_{\text {in }}=T_{m}-0.01 \mathrm{~K}$. Dashed line-nucleation rate in quasi-static conditions, $\dot{T} \tau=0$.

times as long as $10^{2}-10^{5} \mathrm{~s}[5,31,48]$, and in such systems, relaxation does play critical role in the nucleation kinetics.

Transient nucleation rates $\dot{N}_{\text {trans }}(T)$ are the smaller, the larger is the product $|\dot{T} \tau|$. Taking into account that relaxation times in the liquid-solid transitions are in the nanosecond range (Fig. 6) and practically attainable cooling/heating rates do not exceed $10^{6} \mathrm{~K} /$ $\mathrm{s}$, the product $|\dot{T} \tau|$ is smaller than $10^{-3} \mathrm{~K}$. Consequently, effect of relaxation is weak and in most cases can be neglected. When the relaxation time $\tau$ is equal to zero and cooling rate $\dot{T}$ is different from zero, the nucleation process is quasi-static. Then, cluster distribution and nucleation rate follow variation in temperature and in every instant of time assume the steady-state value (dashed lines in Fig. 6)

Figure 7 Athermal nucleation rates $\dot{N}_{a t h}$ versus temperature $T$ for-a indium, $\mathbf{b}$ PHB. Cooling rates $\dot{T}$ indicated. Dashed linesteady-state thermal nucleation rate $\dot{N}_{s t}$.

$$
\begin{array}{r}
\rho_{\text {trans }}[v, T(t)] \stackrel{\tau \rightarrow 0}{\longrightarrow} \rho_{\text {st }}[v, T(t)] \\
\dot{N}_{\text {trans }}[T(t)] \stackrel{\tau \rightarrow 0}{\longrightarrow} \dot{N}_{\text {st }}[T(t)]
\end{array}
$$

Kelton [7, 34] discussed homogeneous crystal nucleation in a metallic glass $\mathrm{Au}_{81} \mathrm{Si}_{19}$ as a function of temperature and cooling rate. Maximum nucleation rate of the order of $10^{18} \mathrm{~cm}^{-3} \mathrm{~s}^{-1}$ in the steady state $(\dot{T}=0)$ is reduced to ca $10^{4} \mathrm{~cm}^{-3} \mathrm{~s}^{-1}$ at $\dot{T}=-10^{7} \mathrm{~K} / \mathrm{s}$. Similar behavior is evident in Fig. 6 where the nucleation rates for indium and PHB plotted with $\dot{T} \tau=$ const are reduced by three orders of magnitude when steady-state conditions $(\dot{T} \tau=0)$ are replaced with $\dot{T} \tau=-10^{4} \mathrm{~K}$.

Figure 7 presents athermal nucleation rates for indium and PHB computed from Eq. (41) as functions of temperature $T$ at constant cooling rates. It is evident that athermal nucleation rate is a function of temperature and cooling rate. Dashed line in each figure presents steady-state thermal nucleation rate, 
Eq. (18). $\dot{N}_{\text {ath }}$ increases with increasing undercooling, $T_{m}-T$, and with increasing cooling rate. Unlike transient nucleation rates (Fig. 6), steady-state thermal nucleation rate does not provide any bound for athermal nucleation. The function $\dot{N}_{\text {ath }}(T)$ starts from zero at $\dot{T}=0$ and approaches infinity at infinite cooling rate.

Steady-state thermal nucleation rate provides boundary for transient nucleation mechanism and not for athermal nucleation. At some cooling rates, $\dot{T}=\dot{T}_{\text {cross }}(T)$, athermal nucleation rate crosses steadystate thermal nucleation rate, and both mechanisms have the same influence on nucleation. For athermal nucleation under quasi-static conditions, we obtain

$$
\begin{array}{r}
\dot{N}_{\text {ath }}\left[T, \dot{T}_{\text {cross }}(T)\right]=\dot{N}_{\text {st }}(T) \\
\dot{T}_{\text {cross }}(T)=\frac{-\Delta h\left(T_{m}-T\right)^{2} \sqrt{k T}}{4 \sqrt{6 \pi} \sigma^{3 / 2} T_{m}} \frac{1}{\tau}
\end{array}
$$

The athermal nucleation overruns the thermal process at the cooling rates exceeding $\dot{T}_{\text {cross }}$ which is inversely proportional to the relaxation time $\tau$ and increases with increasing the undercooling, $T_{m}-T$. The function $\dot{N}_{\text {ath }}(T)$ starts from zero at $\dot{T}=0$ and approaches infinity at infinite cooling rate. With decreasing temperature $(\dot{T}<0)$, the crossover cooling rate and athermal nucleation rate at the crossover, $\dot{N}_{\text {ath }}\left[T, \dot{T}_{\text {cross }}(T)\right]$, predicted from Eq. (48) increase. For example, in the case of indium at $T=337 \mathrm{~K}$, we have $\dot{N}_{\text {ath }}(T)=\dot{N}_{\text {st }}(T)=10^{5} \mathrm{~cm}^{-3} \mathrm{~s}^{-1} \quad$ at $\quad \dot{T}_{\text {cross }}=-1.8 \times$ $10^{11} \mathrm{~K} / \mathrm{s}$. The same nucleation rates for PHB are achieved at $T=427 \mathrm{~K}$ and $\dot{T}_{\text {cross }}=-4.4 \times 10^{10} \mathrm{~K} / \mathrm{s}$. At small undercoolings, the crossover nucleation rates are negligibly small in both cases. At deeper undercoolings, the cooling rates required for the crossover are extremely high due to high thermal nucleation rates exceeding experimentally available range. This behavior predicted for the molten metal (indium) and for the polymer melt (PHB) seems to be typical for liquid-to-solid transitions characterized by very short relaxation times.

Contribution of athermal nucleation (positive or negative) in case of pure sporadic nucleation is negligibly small for experimentally controlled cooling rates, compared to thermal mechanism. Significant contribution of athermal nucleation might be expected in processes with predetermined homogenous nuclei, for example memory effects, which modify the cluster size distribution.
Total concentration of nuclei created in a single cooling/heating run is the sum of integrals of thermal nucleation rate reduced by cooling/heating rate (Eq. 43), and athermal contribution controlled by temperature interval $\left(T_{0}-T\right)$ but independent of the cooling/heating rate (Eq. 45). Thermal nucleation mechanism provides positive contribution to the concentration of nuclei both for cooling and heating. Athermal nucleation, on the other side, yields positive contribution at cooling and negative at heating.

Figure 8 illustrates the concentration of nuclei $\Delta N_{q s}(T)$ produced in a quasi-static process during cooling at various cooling rates for indium and PHB, calculated from Eq. (46) at the initial temperature $T_{0}=T_{m}-0.01 \mathrm{~K}$ (dashed lines). Solid lines in Fig. 8 show a gain in the concentration of nuclei due to the athermal quasi-static process, $\Delta N_{\text {ath }}(T)$. At higher cooling rates, lower increase in the concentration of nuclei produced in thermal mechanism is obtained at
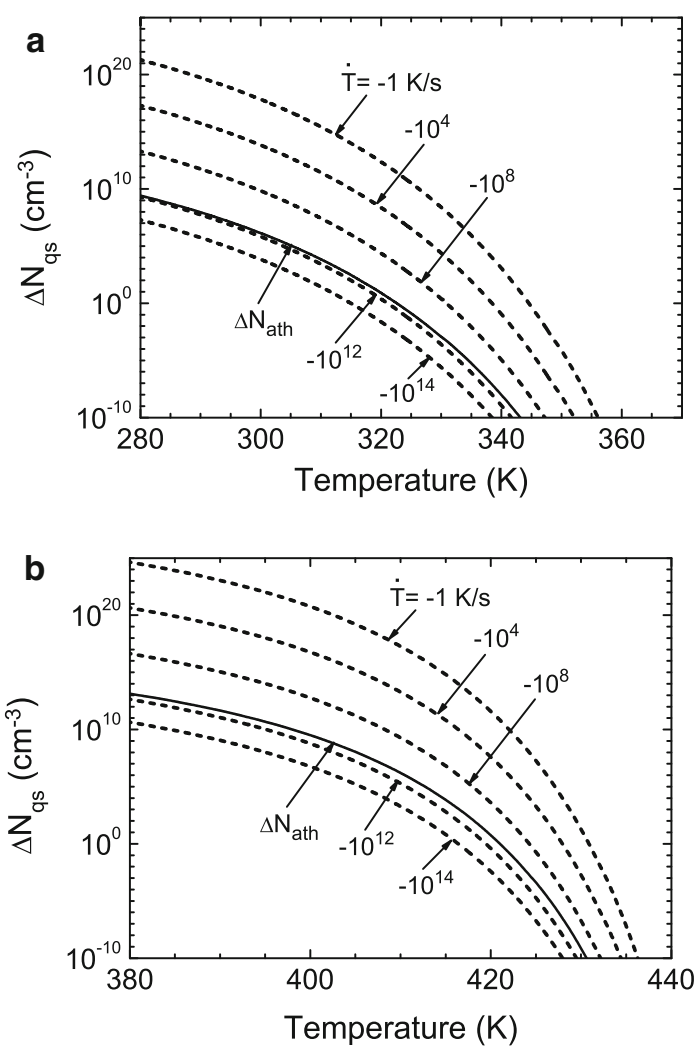

Figure 8 Concentration of nuclei produced in quasi-static process $\Delta N_{q s}$ versus temperature $T$ (dashed lines) at various cooling rates (indicated) for-a indium, b PHB. Solid line-gain in the nuclei concentration due to athermal nucleation. $T_{0}=T_{m}-0.01 K$. 
any temperature $T$, while concentration of athermal nuclei created is independent of the cooling rate.

\section{Effects of material properties}

Two model materials: molten elemental indium and molten organic polymer (PHB) are discussed. The material characteristics are collected in Table 1. In spite of different molecular structures, kinetics of nucleation behavior is qualitatively similar for both materials. Basic differences between molten indium and PHB involve the size of single kinetic units, density, heat of melting, equilibrium melting temperature, molecular mobility, and interface free energy density.

For indium, the kinetic units considered are single atoms, and for PHB statistical Kuhn segments with the volume ca 100 times larger than indium atoms. Although heat of fusion of indium is $13 \%$ greater than that of $\mathrm{PHB}$, its melting temperature is lower by about $40 \mathrm{~K}$ than that of PHB. Melting entropy of the polymeric material is smaller because of some intramolecular order in the molten state. Molecular mobility of PHB approximated by the Arrhenius formula is much smaller than that of indium due to the activation energy $E_{D}$ nearly six times higher for the polymer. Interface free energy densities, nearly twice higher for indium, affect thermodynamic barrier of nucleation, $\Delta G^{*}$.

Nucleation rates start as zero at the respective melting temperatures $T_{m}$ and increase with increasing undercooling, $\Delta T=T_{m}-T$. To obtain realistic homogeneous nucleation rates (say, $1 \mathrm{~cm}^{-3} \mathrm{~s}^{-1}$ ), indium should be undercooled by about $80 \mathrm{~K}$ below $T_{m}$, while PHB requires twice lower undercooling, about $40 \mathrm{~K}$. The highest nucleation rates calculated for the considered materials approach $10^{22}$ $10^{26} \mathrm{~cm}^{-3} \mathrm{~s}^{-1}$.

Free energy barrier of primary homogeneous nucleation $\Delta G^{*}$ at undercooling $\Delta T$ is influenced by the interface free energy density $\sigma$ and the ratio $\Delta h / T_{m}$ which is the specific entropy of fusion, $\Delta s$. Higher value of $\sigma$ for indium (Table 1) leads at any undercooling $\Delta T$ to higher free energy barrier $\Delta G_{I n}^{*}$ despite higher $\Delta h / T_{m}$. At the undercooling $\Delta T$, the ratio of free energy barriers of indium and $\mathrm{PHB}$

$\frac{\Delta G_{\mathrm{In}}^{*}(\Delta T)}{\Delta G_{\mathrm{PHB}}^{*}(\Delta T)}=\left(\frac{\Delta s_{\mathrm{PHB}}}{\Delta s_{\mathrm{In}}}\right)^{2}\left(\frac{\sigma_{\mathrm{In}}}{\sigma_{\mathrm{PHB}}}\right)^{3}$

For the parameters listed in Table 1, we have $\Delta s_{\mathrm{PHB}} / \Delta s_{\text {In }}=0.809, \sigma_{\mathrm{In}} / \sigma_{\mathrm{PHB}}=1.872$, and $\Delta G_{\mathrm{In}}^{*}(\Delta T) /$ $\Delta G_{\mathrm{PHB}}^{*}(\Delta T)=4.29$ at any undercooling. The transport activation energy is considerably lower for indium, $E_{D}^{\mathrm{In}} / E_{D}^{\mathrm{PHB}}=0.180$, and reduces the nucleation kinetics much less than for the polymer. Considerably lower volume of single elements for indium, $v_{0}^{\text {In }} / v_{0}^{\mathrm{PHB}}=1.115 \times 10^{-2}$, leads to much higher preexponential factor $I_{0}$ in Eq. (18), enhanced by higher $\sigma_{I n}$ in the power of $1 / 2$.

Analysis of individual influence of $\sigma, \Delta h / T_{m}, E_{D}$, and $v_{0}$ indicates that despite much higher pre-exponential and transport terms for indium, the steadystate nucleation rate, Eq. (18), is lower than that for PHB by many orders of magnitude due to higher value of $\sigma$ for indium. Higher $\sigma$ results in steadystate nucleation rate for indium lower by 22 orders of magnitude at high undercooling $(\Delta T=100 \mathrm{~K})$. With decreasing undercooling, the reduction in the nucleation rate increases, and for $\Delta T=35 \mathrm{~K}$, it reduces by 151 orders of magnitude. The effect of higher values of $\Delta h / T_{m}$ for indium is the steady-state nucleation rate higher by nine orders of magnitude at $\Delta T=$

Table 1 Material characteristics of indium and polyhydroxybutyrate (PHB)

\begin{tabular}{llllll}
\hline Material characteristics & Indium & & PHB \\
\hline$T_{m}(\mathrm{~K})$ & Melting temperature & 429.80 & {$[54]$} & 470.0 & {$[55]$} \\
$\Delta h\left(\mathrm{~J} / \mathrm{cm}^{3}\right)$ & Heat of fusion per unit volume & 209.20 & Calculated from [54, 56] & 185.0 & Calculated from [55] \\
$\Delta h / \gamma(\mathrm{J} / \mathrm{g})$ & Heat of fusion per unit mass & 28.62 & {$[54]$} & 146.0 & {$[55]$} \\
$\sigma\left(\mathrm{J} / \mathrm{cm}^{2}\right)$ & Interface free energy density & $2.730 \times 10^{-6}$ & Calculated from [7] & $1.458 \times$ & Calculated from [50] \\
& & & & $10^{-6}$ & \\
$E_{D}$ & Activation energy & 4.39 & Calculated from [57] & 24.36 & {$[58]$} \\
$(\mathrm{kJ} / \mathrm{mole})$ & & & & & \\
$d_{0}(\mathrm{~cm})$ & Average dimension of kinetic element & $2.97 \times 10^{-8}$ & Calculated from [56] & $1.33 \times 10^{-7}$ & Calculated from [55, 59] \\
$v_{0}\left(\mathrm{~cm}^{3}\right)$ & Volume of kinetic element & $2.62 \times 10^{-23}$ & {$[56]$} & $2.35 \times 10^{-21}$ & {$[55,59]$} \\
$\gamma\left(\mathrm{g} / \mathrm{cm}^{3}\right)$ & Crystal density & 7.31 & {$[56]$} & 1.26 & {$[55]$} \\
\hline
\end{tabular}


$100 \mathrm{~K}$ which increases with decreasing undercooling to 70 orders of magnitude at $\Delta T=35 \mathrm{~K}$. This indicates that higher $\Delta h / T_{m}$ for indium does not compensate much higher reduction in thermal nucleation kinetics caused by higher $\sigma$. Lower values of $E_{D}$ and $v_{0}$ for indium only slightly rise steady-state nucleation rate by three orders of magnitude and by a factor of 20, respectively, in the discussed range of undercooling.

The ratio of the relaxation time of indium and PHB, important for the transient effects (Eq. 29), at the undercooling $\Delta T$ reads

$$
\begin{aligned}
\frac{\tau_{\text {In }}(\Delta T)}{\tau_{\mathrm{PHB}}(\Delta T)}= & \frac{\sigma_{\mathrm{In}}}{\sigma_{\mathrm{PHB}}}\left(\frac{\Delta s_{\mathrm{PHB}}}{\Delta s_{\text {In }}}\right)^{2}\left(\frac{v_{0}^{\mathrm{PHB}}}{v_{0}^{\mathrm{In}}}\right)^{4 / 3} \\
& \times \exp \left[\frac{E_{D}^{\mathrm{In}}}{k\left(T_{m}^{\mathrm{In}}-\Delta T\right)}-\frac{E_{D}^{\mathrm{PHB}}}{k\left(T_{m}^{\mathrm{PHB}}-\Delta T\right)}\right]
\end{aligned}
$$

The value of $E_{D}$ lower for indium by about six times makes the relaxation time by three orders of magnitude lower than for the polymer. The interface free energy $\sigma$ contributes to $\tau$ linearly and does not have such a dominant influence as it has on steady-state nucleation rate. Much smaller volume $v_{0}$ for indium results in high value of 498 of the pre-exponential factor in Eq. (50). Minor influence of $\sigma$ and $\Delta h / T_{m}$ on the difference in the relaxation time of indium and PHB is predicted. Lower $E_{D}$ and $v_{0}$ values for indium compensate each other in the influence on the difference in the relaxation time of both materials. The estimated ratio $\tau_{\mathrm{In}}(\Delta T) / \tau_{\mathrm{PHB}}(\Delta T)$ varies between slightly below unity at high undercooling and about 3 at low undercooling. This indicates similarity of the transient effects in the nucleation kinetics in a wide range of temperature for both materials.

The ratio of athermal nucleation rate of both materials at the undercooling $\Delta T$ and temperature rate $\dot{T}$ is given by the ratio of functions $\Phi$ (Eq. 41), $\dot{N}_{\text {ath }}^{\text {In }} / \dot{N}_{\text {ath }}^{\mathrm{PHB}}=\Phi_{\text {In }}\left(T_{m}-\Delta T\right) / \Phi_{\mathrm{PHB}}\left(T_{m}-\Delta T\right), \quad$ and is influenced by $\sigma, \Delta h / T_{m}$ and $v_{0}$. The parameters influence the free energy barrier in $\Phi(T)$ higher for indium mainly due to higher value of $\sigma$ and reduce the athermal nucleation rate ratio by many orders of magnitude. Much lower value of $v_{0}$ for indium results in a high value of the ratio of the pre-exponential factors $\left(\frac{v_{0}^{\mathrm{PHB}}}{v_{0}^{\ln }}\right)^{2}\left(\frac{\sigma_{\mathrm{In}}}{\sigma_{\mathrm{PHB}}}\right)^{3}\left(\frac{\Delta s_{\mathrm{PHB}}}{\Delta \Delta_{\mathrm{In}}}\right)^{3}=2.80 \times 10^{4}$ in $\Phi(T)$, which does not compensate strong influence of $\sigma$ on the free energy barrier and the athermal nucleation kinetics. Higher $\sigma$ for indium reduces athermal nucleation rate by 21 orders of magnitude at high undercooling $(\Delta T=100 \mathrm{~K})$, and the reduction increases with decreasing undercooling to 151 orders of magnitude at $\Delta T=35 \mathrm{~K}$. Contrary to the effect of higher value of $\sigma$, higher entropy of fusion $\Delta h / T_{m}$ for indium only partly compensates the influence of $\sigma$ and rises athermal nucleation rate from 9 to 69 orders of magnitude with decreasing undercooling from $\Delta T=100-35 \mathrm{~K}$. Similar influence of higher $\sigma$ and $\Delta h / T_{m}$ of indium is predicted also for steady-state nucleation kinetics.

The data for indium are shifted in the temperature scale by some $70 \mathrm{~K}$ with respect to PHB (Figs. 1, 2). Compared at constant temperature, or at constant undercooling, homogeneous nucleation rates are higher for PHB because of higher thermodynamic driving force of crystallization. At the same time, experimental observations for indium [49] show crystallization onset at the undercooling as low as $10-25 \mathrm{~K}$. This effect has been identified as heterogeneous nucleation on the bulk impurities. According to the authors [49], much deeper undercooling is required for homogeneous nucleation. In a bacterial PHB free from impurities, Barham [50] observed homogeneous crystal nucleation under high undercoolings.

Summing up, kinetics of nucleation in molten indium and PHB are similar and seem to be representative of metals, polymers, and other materials undergoing liquid-solid transition. Different behavior has been observed in glass-to-solid and solid-tosolid transitions [7, 31].

\section{Effects of processing conditions}

Nucleation in the liquid-solid transitions is controlled by the thermodynamic variables (temperature, pressure, etc.) and variation rates of these variables (cooling/heating rate, compression rate, etc.). The thermodynamic potentials as functions of the variables determine phase equilibria and the range of conditions in which individual transitions take place. Undercooling determines thermodynamic driving force of the transition, $\Delta g(T)=-\Delta h$ $\left(T_{m}-T\right) / T_{m}$, affects the rate of cluster formation, and enhances nucleation rates and the relaxation phenomena. The rates of temperature, pressure, and other functions of state play important role in the kinetics of nucleation. In this paper, we have 
concentrated on the effects of temperature and temperature rate in isobaric systems.

Nucleation rate is controlled by the competition of thermodynamic influence of undercooling and molecular mobility of the kinetic units subjected to clustering. The measure of molecular mobility is temperature-dependent diffusion coefficient in the space of cluster volumes, $D(v, T)$. In this paper concerned with nucleation in a liquid phase, the Arrhenius approximation was used, $D(v, T) \propto \exp$ $\left(-E_{D} / k T\right)$ with a constant transport barrier, $E_{D}$. For polymers subjected to deeper undercooling, close to glass transition $T_{g}$, the Arrhenius approximation may be replaced with Williams-Landel-Ferry formula [51] $D(v, T) \propto \exp \left[\frac{C_{1}\left(T-T_{g}\right)}{C_{2}+T-T_{8}}\right]$ or Hoffman-Lauritzen formula [3], $D(v, T) \propto \exp \left[\frac{-E_{D}}{k\left(T-T_{\infty}\right)}\right]$, where $T_{\infty}=T_{g}-C_{3}$ and $C_{1}, C_{2}, C_{3}$ are material constants.

The model indicates how the processing conditions can be adjusted to produce desired nucleation structure. Consider conditions required to minimize the concentration of nuclei to get nearly amorphous material. (1) The starting structure should be amorphous. This requires superheating at a temperature $T_{0}>T_{m}$ long enough to erase structural memory $[52,53]$. (2) The cooling rate $\dot{T}$ should be adjusted as high as possible. Complete elimination of the production of thermal nuclei is not possible as it would require infinite cooling rate. (3) The target temperature, $T$, should be adjusted according to the material involved. $T$ must be higher than limiting level, $T>T_{\text {lim }}$ (Eq. 7). For a given material characterized by steady-state nucleation rate $\dot{N}_{s t}(T)$ (cf. Figs. 1, 2), the target temperature should be chosen so that production of thermal nuclei is minimized (see Eq. 43). (4) In addition to thermal nucleation, cooling of a superheated melt provides concentration of athermal nuclei independent of cooling rate and increasing with the temperature interval $T_{0}-T$ (see Eq. 45). Ideal amorphous structure without any nuclei below $T_{m}$ cannot be obtained for two reasons: finite cooling rate and athermal nucleation.

Maximization of the concentration of homogeneous (thermal and athermal) nuclei can be performed according to a similar scheme. The starting temperature $T_{0}$, cooling (heating) rate, and the initial structure are immaterial. One can produce nuclei by cooling superheated amorphous melt, or by heating partly nucleated material to the target temperature.
The fastest way consists in cooling or heating the material to the temperature corresponding to the maximum nucleation rate (Figs. 1, 2). At the target temperature, further nucleation is performed isothermally. In the conditions when thermal and athermal nucleation does not provide expected concentration of nuclei, heterogeneous nuclei can be added to the system.

\section{Conclusions}

A kinetic model of homogeneous non-isothermal crystal nucleation with transient and athermal effects is developed. Transient effects are characterized by a single (the longest) relaxation time. Closed-form analytical formulas for transient cluster size distribution, related thermal and athermal nucleation rates, and the total number of nuclei produced in a cooling or heating run are derived. Under isothermal conditions, the transient term controlled by the relaxation time contributes to nucleation rate the more the higher is temperature. The longer is relaxation time and the smaller is undercooling, the more isothermal nucleation rate lags behind the steady state (Figs. 3, 4).

Under non-isothermal conditions, relaxation effects in thermal nucleation rate are controlled by the product of cooling (heating) rate and temperature-dependent relaxation time, $\dot{T} \tau$. The cooling rate $\dot{T}$ and relaxation time $\tau$ affect nucleation kinetics in a similar way, the longer is $\tau$ and/or the faster is cooling, the closer is nucleation rate to the initial value. Under slow cooling and/or short relaxation times, nucleation rate approaches asymptotically steady state (Fig. 6). Nucleation rate is reduced by relaxation effects the more, the longer is relaxation time and the faster is cooling.

Our model applied to molten indium and the linear polymer $\mathrm{PHB}$, representing molten metals and polymers undergoing liquid-to-solid transitions, are characterized by relaxation times in the nanosecond range. This indicates that transient effects in nucleation would require extremely fast cooling of such materials. Material parameters strongly affecting kinetics of homogeneous nucleation include interface free energy density $\sigma$ and the ratio $\Delta h / T_{m}$. The highest impact on the nucleation rates results from considerably higher $\sigma$ for indium which reduces steady-state and athermal nucleation rates by many 
orders of magnitude, relative to those predicted for the polymer. The reduction is stronger at lower undercooling. Higher value of $\Delta h / T_{m}$ for indium results in a rise of the nucleation rates by some orders of magnitude, but it does not compensate the reduction caused by the $\sigma$ value.

The predicted relaxation time $\tau$ is of the same order of magnitude for indium and PHB in a wide range of undercooling, indicating similarity of the transient effects for both materials. Minor influence of higher values of $\sigma$ and $\Delta h / T_{m}$ for indium on the relaxation time $\tau$ is predicted, while lower values of $E_{D}$ and $v_{0}$ for indium compensate each other.

Total concentration of nuclei created in a single cooling or heating run is a sum of an integral of thermal nucleation rate reduced by temperature rate (Eq. 43) and the athermal contribution dependent on temperature interval $\left(T_{0}-T\right)$. The athermal contribution is independent of the cooling/heating rate (Eq. 45). Thermal nucleation provides a positive contribution to the concentration of nuclei, both for cooling and heating. Athermal nucleation, on the other side, yields positive contribution at cooling and negative one at heating.

Our model can be used to adjust processing conditions for creation desired nucleation structure. It should be noted that ideal amorphous structure free of nuclei cannot be obtained for two reasons: limited cooling rate and athermal nucleation.

\section{Acknowledgements}

This work was supported by the basic funds of the Institute of Fundamental Technological Research, Polish Academy of Sciences for the scientific research.

\section{Compliance with ethical standards}

Conflict of interest The authors declare that they have no conflict of interest.

Open Access This article is distributed under the terms of the Creative Commons Attribution 4.0 International License (http://creativecommons.org/ licenses/by/4.0/), which permits unrestricted use, distribution, and reproduction in any medium, provided you give appropriate credit to the original author(s) and the source, provide a link to the Creative Commons license, and indicate if changes were made.

\section{References}

[1] Frenkel J (1946) Kinetic theory of liquids. Oxford University Press, London

[2] Zettlemoyer AC (ed) (1969) Nucleation. Marcel Decker, New York

[3] Hoffman JD, Davis GT, Lauritzen JI Jr (1976) The rate of crystallization of linear polymers with chain folding. In: Hannay NB (ed) Treatise on solid state chemistry, vol 3. Plenum Press, New York, pp 598-600

[4] Kelton KF (1991) Crystal nucleation in liquids and glasses. Solid State Phys 45:75-177

[5] Kashchiev D (2000) Nucleation. Butterworth-Heinemann, Oxford

[6] Kelton KF, Greer AL (2010) Nucleation in condensed matterapplications in materials and biology. Elsevier, Amsterdam

[7] Kelton KF (2013) Crystal nucleation in supercooled liquid metals. Int J Microgravity Sci Appl 30:11-18

[8] Volmer M, Weber E (1926) Nucleus formation in supersaturated systems. Z Phys Chem 119:277-301

[9] Farkas L (1927) Keimbildungsgeschwindigkeit in übersättigten Dämpfen. Z Phys Chem (Leipzig) A125:236-242

[10] Becker R, Döring W (1935) Kinetic treatment of grain formation in supersaturated vapours. Ann Physik 24:719-752

[11] Zeldovich YB (1943) On the theory of new phase formation: cavitation. Acta Physicochim URSS 18:1-22

[12] Turnbull D, Fisher JC (1949) Rate of nucleation in condensed systems. J Chem Phys 17:71-73

[13] Eyring H (1935) The activated complex in chemical reactions. J Chem Phys 3:107-115

[14] Lauritzen JI, Hoffman JD (1960) Theory of formation of polymer crystals with folded chains in dilute solution. J Res Nat Bur Std 64A:73-102

[15] Hoffman JD, Lauritzen JI (1961) Crystallization of bulk polymers with chain folding: theory of growth of lamellar spherulites. J Res Nat Bur Std 65A:297-336

[16] Ziabicki A (1968) Generalized theory of nucleation kinetics. I. General formulations. J Chem Phys 48:4368-4374

[17] Cormia RL, Price FP, Turnbull D (1962) Kinetics of crystal nucleation in polyethylene. J Chem Phys 37:1333-1340

[18] Burns JR, Turnbull D (1966) Kinetics of crystal nucleation in molten isotactic polypropylene. J Appl Phys 37:4021-4026

[19] Turnbull D (1950) Formation of crystal nuclei in liquid metals. J Appl Phys 21:1022-1028

[20] Turnbull D (1952) Kinetics of solidification of supercooled liquid mercury droplets. J Chem Phys 20:411-424

[21] Collins FC (1955) Time lag in spontaneous nucleation due to non-steady state effects. Z Elektrochem 59:404-407

[22] Kashchiev D (1969) Solution of the non-steady state problem in nucleation kinetics. Surf Sci 14:209-220 
[23] Chakraverty BK (1966) Kinetics of clustering process. Surf Sci 4:205-220

[24] Wakeshima H (1954) Time lag in the self-nucleation. J Chem Phys 22:1614-1615

[25] Feder J, Russel KC, Lothe J, Pound GM (1966) Homogeneous nucleation and growth of droplets in vapors. Adv Phys 15:111-178

[26] Andres RP, Boudart M (1965) Time lag in multistate kinetics: nucleation. J Chem Phys 42:2057-2064

[27] Kelton KF, Greer AL, Thompson CV (1983) Transient nucleation in condensed systems. J Chem Phys 79:6261-6276

[28] James PF (1974) Kinetics of crystal nucleation in lithium silicate glasses. Phys Chem Glasses 15:95-105

[29] Luborsky FE (1977) Crystallization of some Fe Ni metallic glasses. Mater Sci Eng 28:139-144

[30] Scott MG (1978) The crystallization kinetics of Fe-Ni based metallic glasses. J Mater Sci 13:291-296

[31] James PF (1985) Kinetics of crystal nucleation in silicate glasses. J Non-Cryst Solids 73:517-540

[32] Kalinina AM, Filipovich VN, Fokin VM (1980) Stationary and nonstationary crystal nucleation rate in a glass of $2 \mathrm{Na}_{2} \mathrm{O}-\mathrm{CaO}-3 \mathrm{SiO}_{2}$ stoichiometric composition. J NonCryst Solids 38-39:723-728

[33] Kelton KF, Greer AL (1988) Test of classical nucleation theory in a condensed system. Phys Rev B 38:10089

[34] Kelton KF, Greer AL (1986) Transient nucleation effects in glass formation. J Non-Cryst Solids 79:295-309

[35] Kelton KF (1993) Numerical model for isothermal and nonisothermal crystallization of liquids and glasses. J Non-Cryst Solids 163:283-296

[36] Kelton KF (1995) Transient nucleation in glasses. Mater Sci Eng B32:145-151

[37] Shen YT, Kim TH, Gangopadhyay AK, Kelton KF (2009) Icosahedral order, frustration, and the glass transition: evidence from time-dependent nucleation and supercooled liquid structure studies. Phys Rev Lett 102:057801

[38] Jarecki L (1991) Diffusion of single elements and steric limitations in kinetic theory of nucleation and crystallization. Coll Polym Sci 269:11-27

[39] Jarecki L (1994) Effects of translational and rotational diffusion on the association in kinetic model of nucleation. Coll Polym Sci 272:784-796

[40] Fisher JC, Hollomon JH, Turnbull D (1948) Nucleation. J Appl Phys 19:775-784

[41] Ziabicki A (1968) Generalized theory of nucleation kinetics. II. Athermal nucleation involving spherical clusters. J Chem Phys 48:4374-4380

[42] George HH (1985) Spinline crystallization of polyethylene terephthalate. In: Ziabicki A, Kawai H (eds) High-speed fiber spinning. Wiley, New York, pp 271-294
[43] Gibbs JW (1961) The Scientific Papers of J. Willard Gibbs, vol. 1. Thermodynamics. Dover Publications Inc., New York

[44] Cahn JW, Hilliard JE (1958) Free energy of nonuniform system. I. Interfacial free energy. J Chem Phys 28:258-267

[45] Ziabicki A, Jarecki L (1978) Theoretical analysis of oriented and non-isothermal crystallization. III. Kinetics of crystal orientation. Colloid Polym Sci 256:332-342

[46] Ziabicki A (1996) Crystallization of polymers in variable external conditions. Part 1. General equations. Colloid Polym Sci 274:209-217

[47] Ziabicki A (1999) Crystallization of polymers in variable external conditions. Part 4. Isothermal crystallization in the presence of variable tensile stress or hydrostatic pressure. Colloid Polym Sci 277:752-761

[48] Gutzov I, Toschev S, Marinov M, Popov E (1968) Mechanismus der Nichtstationaeren Keimbildung und des Kristallwachstums in einem Modellglase. Kristall Technik $3: 337-354$

[49] Zhong J, Jin ZH, Lu K (2001) Melting, superheating and freezing behaviour of indium interpreted using a nucleationand-growth model. J Phys-Condens Mat 13:11443-11452

[50] Barham PJ (1984) Nucleation behavior of poly-3-hydroxybutyrate. J Mater Sci 19:3826-3834

[51] Williams ML, Landel RF, Ferry JD (1955) Temperature dependence of relaxation mechanisms in amorphous polymers and other glass forming liquids. J Am Chem Soc 77:3701-3707

[52] Ziabicki A, Alfonso GC (1994) Memory effects in nonisothermal crystallization. I. Theory. Colloid Polym Sci 272:1027-1042

[53] Supaphol P, Spruiell JE (2000) Crystalline memory effects in isothermal crystallization of syndiotactic polypropylene. J Appl Polym Sci 75:337-346

[54] Sarge SM, Höhne GWH, Cammenga HK, Eysel W, Gmelin E (2000) Temperature, heat and heat flow rate calibrations of scanning calorimeters in the cooling mode. Thermochim Acta 361:1-20

[55] Barham PJ, Keller A, Otun EI, Holmes PA (1984) Crystallization and morphology of a bacterial thermoplastic: poly-3hydroxybutyrate. J Mater Sci 19:2781-2794

[56] www.webelements.com/indium. Accessed 9 Nov 2015

[57] Cheng SJ, Bian XF, Zhang JX, Qin XB, Wang ZH (2003) Correlation of viscosity and structural changes of indium melt. Mater Lett 57:4191-4195

[58] El-Hadi Abdel Ghaffar AM (2002) Development of a biodegradable material based on poly (3-hydroxybutyrate) PHB. PhD Dissertation, University of Halle

[59] Beaucage G, Rane S, Sukumaran S, Satkowski MM, Schechtman LA, Doi Y (1997) Persistence length of isotactic poly(hydroxy butyrate). Macromolecules 30:4158-4162 